\title{
Quelques Propriétés du Fer électrobévé
}

\author{
Par M. Auguste BOUCHAYER, Ingénieur des Arts et Manufactures.
}

Le fer électrobévé est du fer électrolytique obtenu par les Etablissements Bouchayer et Viallet qui possèdent, pour la France, les licences de la Société "Le Fer ".

Celte labrication datant d'une dizaine d'années déjà, on songea pendant la guerre à l'utilisation de ce nouveau métal dans les déments de Munitions.

En 1915, sur la demande de M. le général Dumézil, Mr. Portevin, Secretaire général de la Renue de Métallurgie, fit quelques essais mécaniques sur des tôles de fer électrolytique envoyées par les Etablissements Bouchayer et Viallet a l'Atelier de constructions de Puteaux. Mais, en raison de la faible épaisseur de res tôles, plus petite que $0,5 \mathrm{~m} / \mathrm{m}$, les valeurs de l'allongement de rupture trouvées étaient faussées et ne permettaient pas de se faire une idée correcte de la malléabilité de ce produit. C'est avec juste raison que les Cahiers des Charges de l'Artillerie spécilient que pour le laiton spécial en bandes ou en feuilles, il ne sera pas fait d'essais de traction pour les épaisseurs en dessous de $1 \mathrm{~m} / \mathrm{m}$. Latelier de Puteaux ne disposait pas, à cette époque, d'appareils pour l'essai de poinçonnage des tôles qui fournit les meilleures evaluations de la facilite d'emboutissage.

Dans lat suite, en 1916, au moment où on se préoccupait par tous les moyens, de réduire la consommation de cuivre et de ses alliages, on pensa que l'on pourrait avoir recours au fer électrolytique pour la confection de certains éléments de munitions en tôle emboutie exécutés jusqu'alors en laiton.

Il fut utilisé, à ces essais, ce qui restait des échantillons de lôle de 3 dixièmes de millimètres d'épaisseur, pour faire exéculer, grâce à l'obligeance de la Compagnie des Compteurs, divers essais d'emboutissage, notamment de queue de bouchons de gaines-relais.

Les rẹsultats ont dé très satisfaisants ; les figures 1,2 et 3 donnent des photographies des pièces embouties ainsi obtenues par M. Portevin. La figure 1 représente des emboutis obtenus en une seule passe ; les figures 2 et 3 montrent la série complète des opérations pour la queue de bouchons, jusques, y compris, la soudure et l'étamage.

Cet essai montrait, en outre, qu'il n'y avait, pour ainsi dire, pas de modifications à faire subir à l'outillage pour le passage de l'exécution de la piece de laiton à celle de la pièce en fer électrolytique.

A la suite de ces essais, il fut demandé aux Etablissements Bévé d'envoyer à l'Atelier de Constructions de Puteaux, des feuilles de fer électrolytique pour confectionner des étuis de cartouches à balles, pièces dont l'emboutissage est beaucoup plus complexe; ces essais ont également donné satisfaction au point do vue facilité d'emboutissage.

Enfin, en 1917, M. Portevin avait songé à expérimenter la substitution du fer électrobévé au cuivre pour le ceinturage des obus.

Des anneaux de fer électrolytique ont été envoyés dans ce but par l'usine de Grenoble et se sont parfaitement ceinturés sur obus slo 75 et de 155 . Ces essnis ont été effectués aux Usines linault.

Mais ces expériences n'ont pu ètre poursuivies plus loinl, l'opérateur étant de plus en plus absorbé par les services de l'Aéronautique.

A cotto dernière occasion, il avait procédé à une étude préli- minaire de l'écrouissage du fer électrolytique et du recuit après écrouissage, en se servant pour cela des barrettes obtenues en déployant les anneaux ceintures d'obus de 74 ; ils avaient $4 \mathrm{~m}_{i} \mathrm{~m}$ d'épaisseur et $8 \mathrm{~m} / \mathrm{m}$ de largeur.

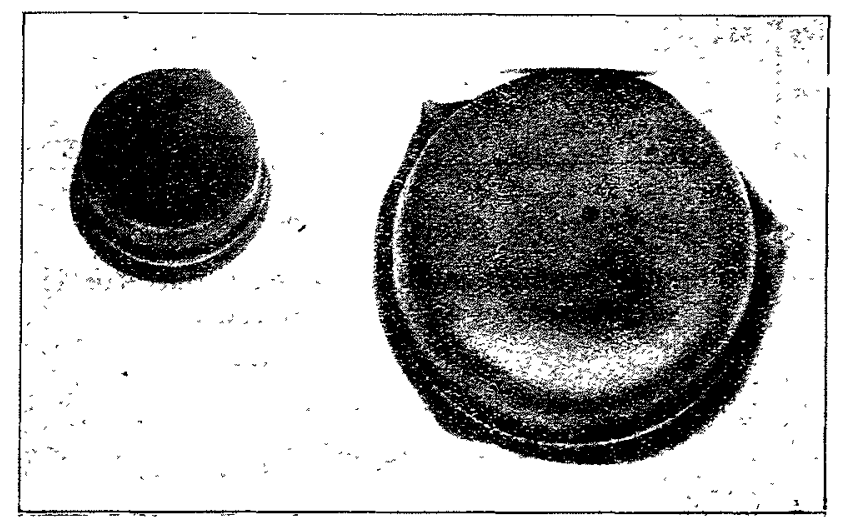

Figg. 1. - Emboutissage d'éléments de munitions. Pièces obtenues on une seule passe.

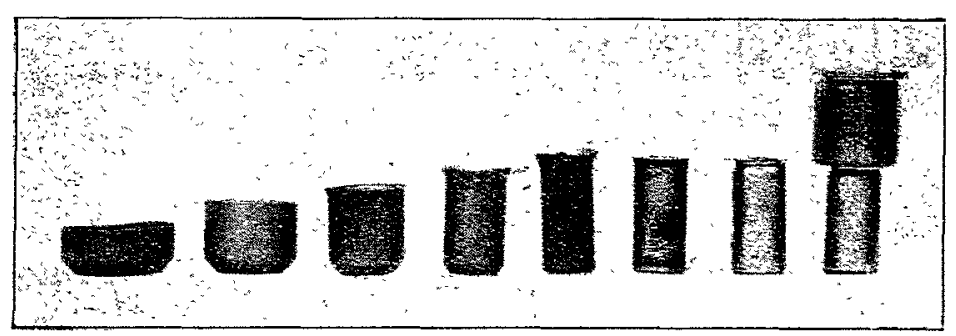

Fig. 2. - Emboutissage d'eléments de munitions. Série complète des opérations pour queue de bouchons.

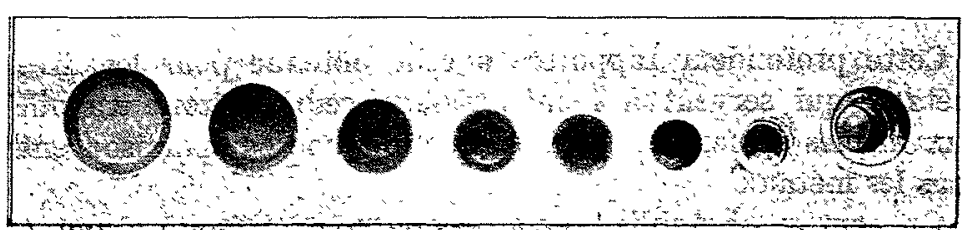

Fig. 3. - Emboutisșage d'éléments de munitions. Sério complète des opérations pour queue de bouchons.

Les résultats sont donnés dans les deux graphiques, figures 4 et 5. L'auteur ajoute qu'ils devraient être vérifies, notamment en ce qui concerne le recuit, en raison surtout de ce qu'il n'a été procédé qu'à une série de déterminations.

Les essais de poinçonnage qui n'avaient pu être faits à Puteaux avaient été entrepris déjà par la Société des Usines de SainteMarie et Gravigny.

Cette demière avait installé dans les locaux des Etablissements Bouchayer et Viallet, un laboratoire pour l'étude des tôles électrolytiques.

Ces essais d'emboutissage furent poursuivis à l'aide de l'appareil Erichsen et portèrent sur un très grand nombre d'échantillons.

La tôle à éprouver est maintenue devant un orifice en face 
duquel une vis micrométrique déplace un poinçon demi-sphérıque de $19 \mathrm{~m} / \mathrm{m}$ de diamètre. Le poinçon avance horizontalement, touche la tôle, puis l'emboutit dans l'orifice. On observe la surface déformée a l'aide d'une petite glace qui permet d'arrèter l'avancement du poinçon au moment précis où le métal commence à cricuuer ; on note alors la profondeur d'emboutissage.

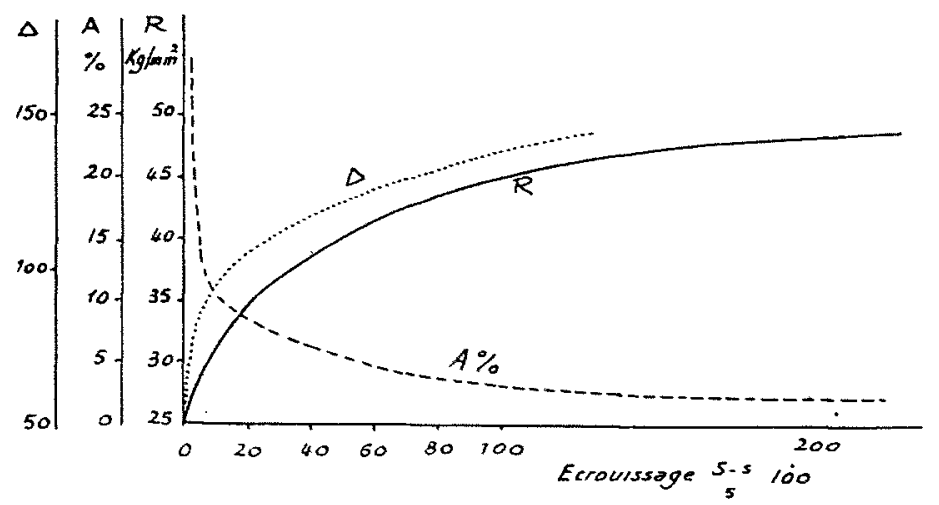

Fig. 4. - Etude de l'écrouissage du fer bévé sur barrette de $4 \mathrm{~m} / \mathrm{m}$ d'épaisseur et $8 \mathrm{~m} / \mathrm{m}$ de largeur.

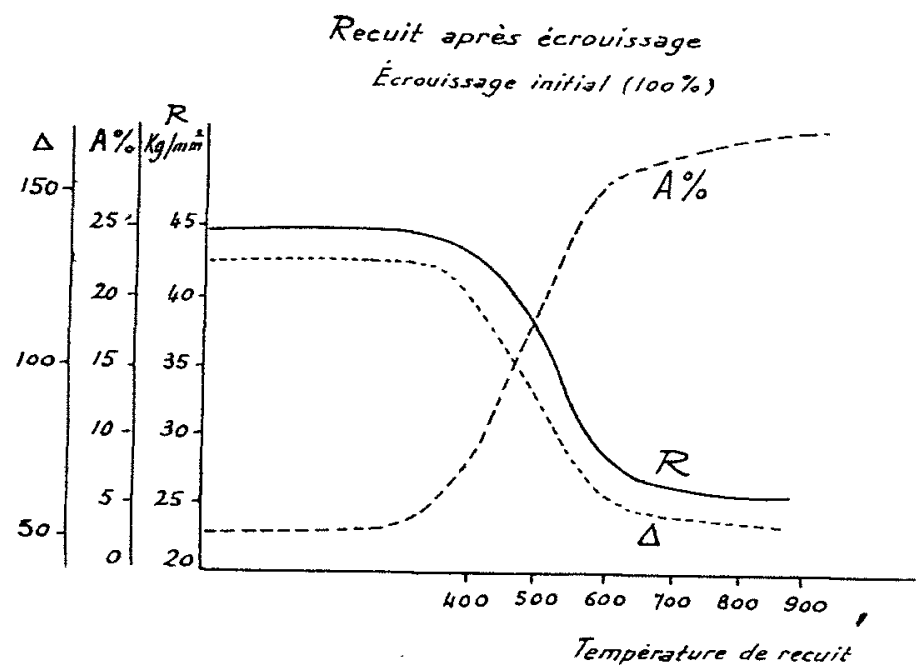

Fiig. 5. - Recuit après écrouissage sur du fer bévé sur barrette de $4 \mathrm{~m} / \mathrm{m}$ d’épaisseur et $8 \mathrm{~m} / \mathrm{m}$ de largeur.

Cette profondeur rapportée à celle obtenue pour les divers métaux qui servent à l'emboutissage, ceux-ci étant travaillé: aux mêmes épaisseurs, permet d'établir une comparaison entre tous les métaux.

On peut aussi établir les courbes de comparaison qui ont été tracées sur le graphique de la figure 6.

Ce graphique donne les courbes des metaux différents. Not: les désignons ci-dessous dans l'ordre de plus grande résistant:* à l'emboutissage, c'est-à-dire dans l'ordre de préférence :

Laiton à emboutir.

Cuivre.

Fer électrolytique.

Fer feuillard blanc.

Aluminium.

Zinc.

Le cuivre et le fer électrobévé sont très rapprochés surtout en allant vers les fortes épaisseurs.

Ce graphique donne aussi les courbes des différentes qualités de fer et d'acier qu'on emploie, en général, pour les travaux d'emboutissage. Je les désigne, comme précédemment, dans l'ordre de qualité :
Fer électrobévé.

Fer feuillard blanc.

Tôle de fer Siemens-Martin à emboutır.

Tôle à plier double décapage.

Tòle à plier simple décapage.

Tôle à plier ordinaire et fer blanc.

Ces résultats expliquent la complète réussite des essais lentés pendant la guerre pour le remplacement du laiton et du cuive rouge.

Ils expliquent également qu'on ait pu entreprendre avec succes des essais de repoussage du métal.

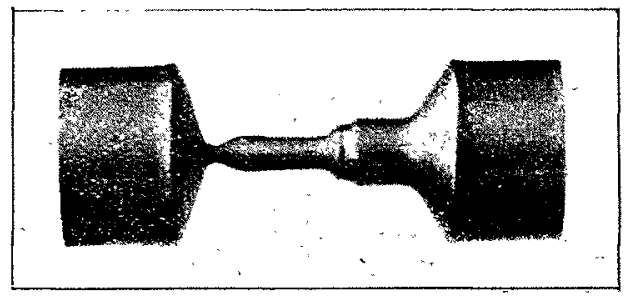

Fig. 7. - Essai de repoussage sur un tube de fer électrobylique de $120 \mathrm{~m} / \mathrm{m}$ de diametre et de $1 \mathrm{~m} / \mathrm{m}$ d'épaisseur.

L'industrie livre des milliers de petits objets, articles parisiens, fabriqués en cuivre rouge, laiton et surtout aluminium, ayant la forme de corps de révolutions. On part généralement d'un flanc qu'on fixe dans un touret qui marche à grande vitesse et à l'aide d'un levier de forme spéciale, on appuie sur le flanc qui plie, se déforme et se moule sur le modèle de l'objet à produire qui est aussi fixé sur le touret. Parfois, on prend le flane avec des pinces, d'autres fois, on travaille a creux sur des tubes pour la confection de petits flacons et d'autres objets.

Etant donné la place qu'occupe le fer électrobévé dans la gamme d'emboutissage, nous l'essayâmes à ce travail de repoussage.

Les résultats obtenus furent concluants. Nous donnons, figure 7, la photographie d'un échantillon de tube travaillé à creux par les procédés que nous venons d'indiquer. Ce tube a un diamètre intérieur de $120 \mathrm{~m} / \mathrm{m}$ et une épaisseur de $1 \mathrm{~m} / \mathrm{m}$. Nous sommes arrivés comme le montre la figure, à réduirc le diamètre à $12^{\mathrm{m} / \mathrm{m}}$ dans sa section la plus réduite, soit dix fois moins que le diamètre primitif.

Le métal s'est refoulé sur lui-mème et son épaisseur a diminué d'autant. Pour arriver a ces résultats extrêmes, on a dù enlever du métal au tour afin de ramener l'épaisseur à des dimensions convenables.

On concevait que cette méthode devait permettre l'exécution de récipients ou corps creux pour gaz comprimés, tout a fait homogènes. c'est-à-dire monoblocs. C'est ce qui a été tenté avec des épaisseurs plus fortes de $2^{\mathrm{m}} / \mathrm{m}$ par exemple.

\section{Eprowué a $140^{\prime}-E p^{\top} 2 \%$}

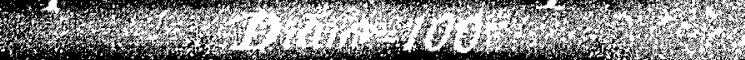
and

Fig. 8. - Bouteille pour gaz sous pression en fer électrobévé de $2 \mathrm{~m} / \mathrm{m}$ d'épaisseur, obtenue sans aucune soudure, par repoussage. 


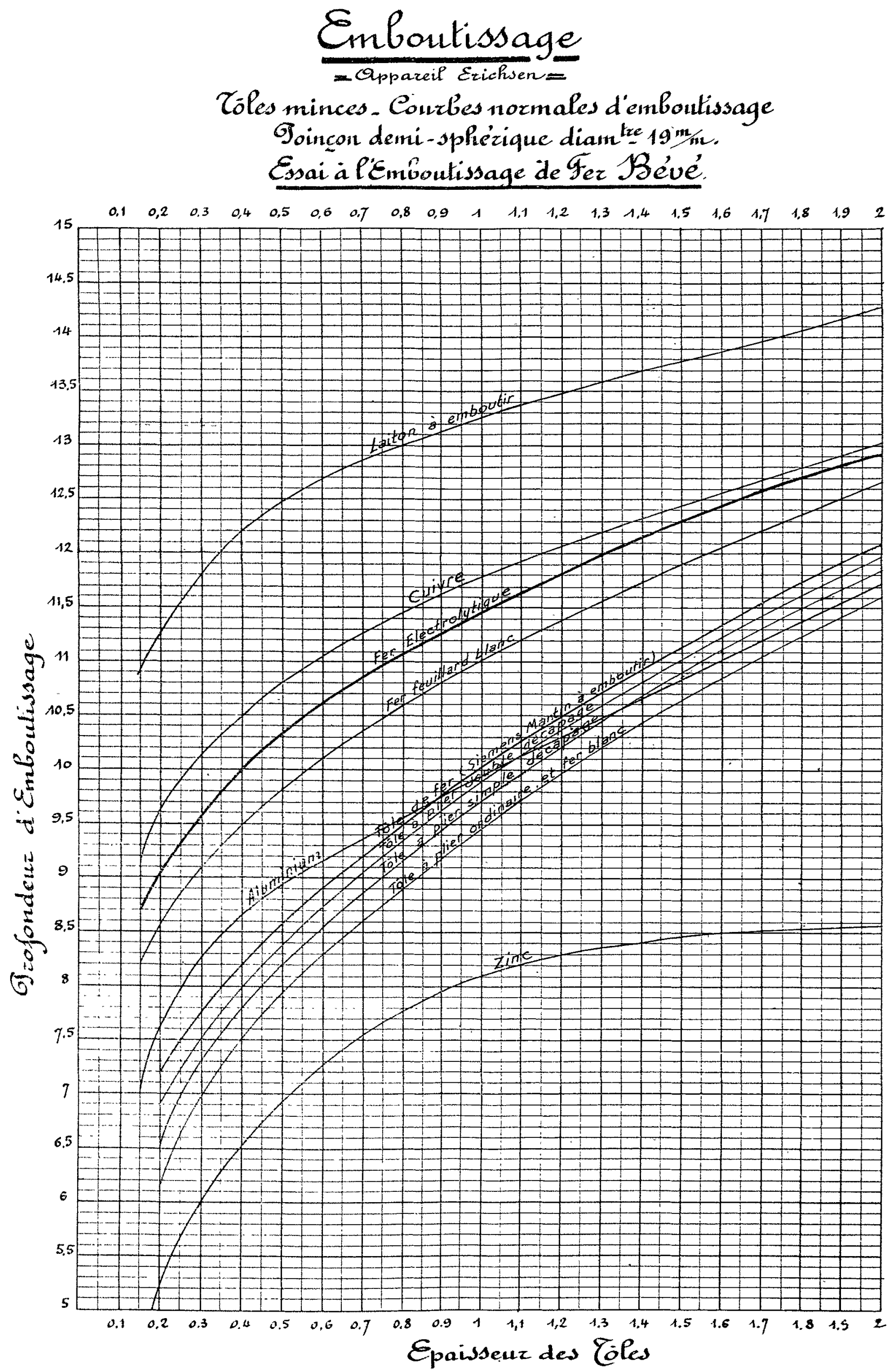

Fìg. 6. - Courbes d'emboutissage des divers métaux, $\mathrm{y}$ compris le fer électrobéve. 
de diametre extérieur. Les deux extrémités du tube ont été repoussés à creux sur un tour. Nous avons été obligés d'emploỵer, comme outil, un lexier à galet de diamètre approprié, le simple levier produisant des grippages.

Le repoussage des extrémités a domné deux fonds, l'un denicylindrique et l'autre conique avec des surépaisseurs telles qu'il etait possible d'obturer l'extrémité avec des bouchons filetés.

L'échantillon représenté a été éprouré à une pression de 140 atmosphères. Les deux extrémités de la bouteille qui avaient étí recuits au maximum pour donner la plus grande ductibilité possible, se sont gonflées sous la pression tandis que la partie médiane légèrement écrouie n’a pas bougé.

D'après la formule de Lamé, le travail du métal avant la déformation des extrémités, était d'environ 32 kilogs par milli mitre carré de section, ce qui correspond bien aux caractéristiques du fer électrolytique.

Ancune déformation permanente n'a été observée dans la partic centrale de la bouteille non recuite.

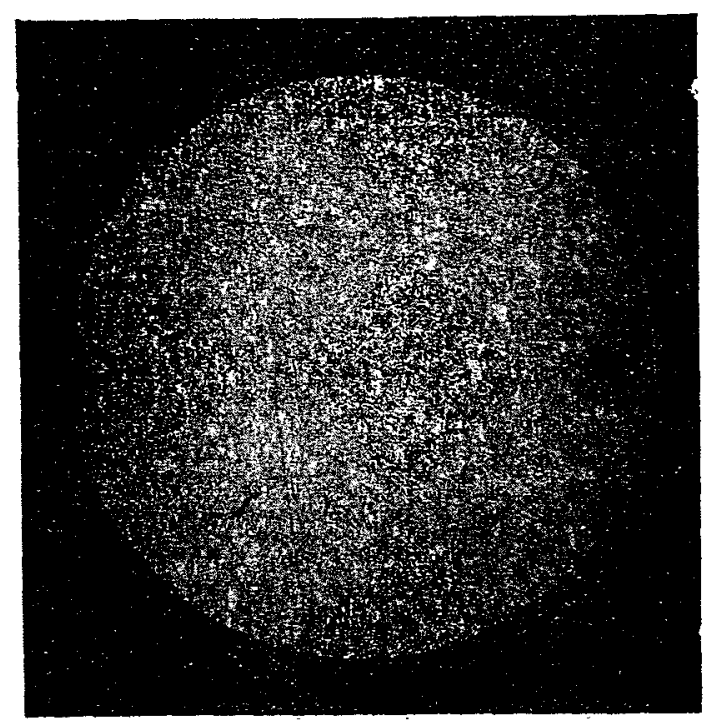

Fig. 9. - Essai micrographique. Structure fibreuse du fer bévé jusqu'à 860 degrés.

En somme, cette bouteille pour fluide sous pression correspond aux données suivantes :

Pression d'épreuve : $140 \mathrm{kgs}$.

Volume à la pression atm. : 5 litres.

Poids du récipient : $3 \mathrm{kgs}$ environ.

Etant donné l'homogéneité très grande du métal dont on a la preuve en constatant l'allongement permanent du métal sous la forme d'un accroissement "régulier de diamètre, on peut ainsi compter avec cette bouteille sur une pression de service de $100 \mathrm{kgs}$ par centimètre carré.

Ce procédé de repoussage nous a permis d'obtenir des creusets d'analyse qui nous ont été demandés par M. le Professeur Flusin, de la Faculté des Sciences de Grenoble.

Ces creusets doivent servir à la désagrégation des ferro-alliages par les alcalis et notamment par le peroxyde de sodium.

Nous avons fourni au Laboratoire une série de ces creusets de diverses épaisseurs qui avaient été obtenus comme nous l'avons indiqué plus haut en partant de petits flancs. Voici les renseignements intéressants que II. Flusin a bien voulu nous donner à leur sujet.

L'attaque des ferro-alliages s'effectue généralement dans des creusets en nickel ou on fer, qui sont eux-mêmes attaqués plus ou moins profondément au cours de la désagregation. Ces creusets ne peuvent servir qu'à un nombre d'opérations limité; de plus, une certaine quantité de métal du creuset et les impuretés du métal se retrouvent dans le produit de l'attaque.

Pour certains ferro-alliages, en particulier pour les ferro-chromes à haute teneur en chrome, les creusets en nickel doivent etre proserits, car l'oxyde de nickel provocque la decomposition l'unc petite partie du chromate formé, et faussc les rẻsultats du dosago volumetrique. D'une façon générale, l'élimination de l'oxyde de nickel dans le produit de l'attaque est une operation ennuyeus, et assez longue ; enfin, les creusets en nickel contiennent pur[ois asse\% de soufre pour faire rejeter leur emploi lorsqu'il s'agit d'un dosage de soufre.

Les creusets en fer, quoiqu'un peu plus attaquables, seraient presque toujours préférés s'il n'était pas souvent diflicile of parfois presque impossible de s'en procurer ; certaines usines électro-métallurgiques fabriquent elles-mèmes leurs crensets ou leurs capsules, en emboutissant de la tỏle de fer.

On reproche aussi i ces creusets leur impurete. On éprowe

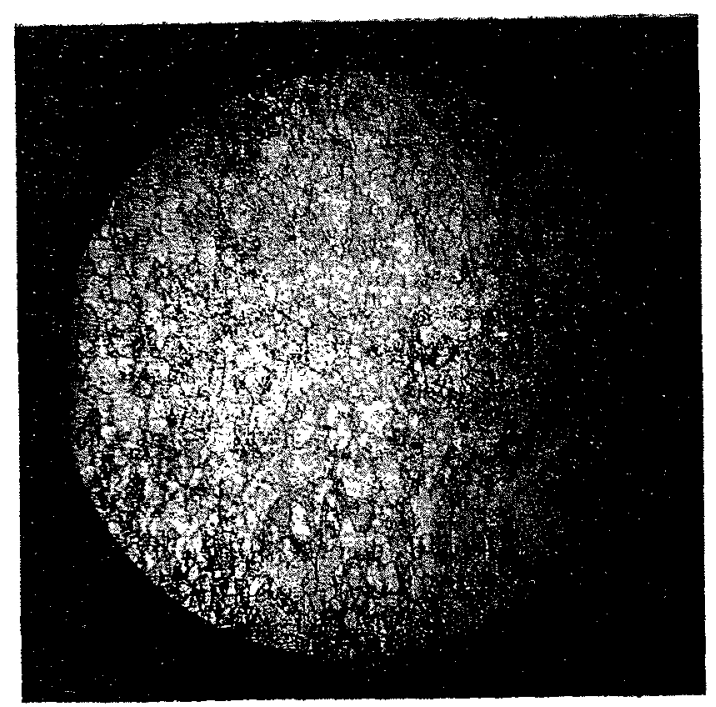

Fig. 10. - Essai micrographique. Structure cristalline du fer bévé après 880 degrés.

parfois au laboratoire des ennuis assez sérieux résultant de la présence, dans le nickel ou dans le fer, de certaines impuretés, telles que soufre, manganèse, etc...

Ces ennuis sont supprimés par l'emploi du fer électrolytique qui, grâce à sa pureté supérieure à celle de tous les fers connus, grâce aussi d̀ sa grande malléabilité, paraît être une matière de choix pour les fabrications des creusets.

Ceux mis à l'essai avaient tous la mème dimension : $45 \mathrm{~m} / \mathrm{m}$ de diamètre supérieur, $25 \mathrm{~m} / \mathrm{ro}$ de diamelre inférieur, et $40^{\mathrm{m} / \mathrm{m}}$ de hauteur. C'est la grandeur courante pour les désagrégations du ferrochrome.

La livraison contenait sept séries correspondantes à des épaisseurs différentes de la paroi ; elles croissaient par 1/10, de 4/10 pour la première série à $10 / 10$ pour la septième sćrie.

les épaisseurs de $4 / 10$ et $5 / 10$ doivent être abondonnees; lo creuset est trop mince et se perce, en cours d'opération, i i i première attaque au peroxyde. L'épaisseur de 6/10 est déjà utilisable, mais celle de 7/10 paraît être l'épaisseur optimum. Les creusets de 7/10 supportenl toujours quatre et souvent cinq désagrégations au peroxyde de sodium, ce qui correspond au service normal d'un creuset en fer ordinaire de même épaisseur.

Avec les creusets de $8 / 10$ a $10 / 10$, nous avons constaté quc l'attaque était trop lente ; mais cet inconvénient est imputable à la très faible pression du gaz d'éclairage à Grenoble, et il est 
probable que dans les laboratoires ou dans les villes ou le gaz est distribué à une pression normale, les, creusets de 8 à 10/10 scraient utilisables.

Lin somme, les creusels de $7 / 10$ domnent toute satisfaction et le ferbévé paraît tout à lait recommandable.

L'altaque des ferrosilicium par les alcalis exige des creusets de plus grandes dimensions ; ceux dont on se sert mesurent $55^{\mathrm{m} / \mathrm{m}}$ de dianètre supérieur, $30 \mathrm{~m} / \mathrm{m}$ de diamètre inférieur, et $55^{\mathrm{m} / \mathrm{m}}$ de hauteur.

Les essais vont se poursuivre avec la Faculté des Sciences de (irenoble, et il y a tout lieu de penser que les deux propriétés du ferbévé : sa pureté et sa malléabilité, en feront, comme le dit le professeur Flusin, unc matière de choix pour cette nouvelle application.

Toutes les operations mécaniques dont nous avons parlé et que peut supporter le ferbévé, grâce à ses propriété spéciales, demandent rependant que le dit métal ait acquis son état définitif obtenu par un recuil se rapprochant de $900^{\circ}$.

C'est aux environs de cette température que le fer électrobévé jossède ses qualités de malléabilité qui en font un métal particulièrement convenable aux emplois que nous avons décrits.

Dans cet état, la structure du fer électrolytique est cristalline, lout à fait analogue à celle des fers purs obtenus par la grosse sidérurgie. Jans l'état précédent celui-ci, la structure est fibreuse.

Cous avons demandé aux laboratoires des Etablissements bouchayer et Viallet, de rechercher le point de passage entre les deux structures et de déterminer, si possible, à quoi correspond ce point.

Des essais ont été conduits dans ce sens à différentes températures et pendant des temps variant de quelques minutes à trois heures. Il a été constaté ce qui suit :

… Jusqu'à une température voisine de $860^{\circ}$ et quel que soit le temps de chauffe dans les limites indiquées ci-dessus, aucun changement ne se produit dans la structure micrographique du fer électro. Elle reste celle du fer brut aciculaire ou fibreuse. (Yoir figure 9.)

- A une température voisine de $860^{\circ}$ et quel que soit le temps de chauffe. il se produit un certain empâtement des fibres ou aguilles sans qu'il soit possible de deceler encore des cristanx individualisés.

- A unre temperature comprise entre $875^{\circ}$ et $880^{\circ}$, le changement de structure se produit brusquement et instantanément. Nous sommes en présence de gros cristaux de ferrite.(Voir figure 10.)

- Point remarquable : ce point de passage entre la structure fibreuse et la structure cristalline correspond exactement au point de transformation allotropique du fer donné par la courbe de dilatation.

Lin rèsumé, le fer électrolytique Bévé, offre cetle particularité de présenter un point de passige tris net entre la structure fibreuse et la structure eristalline. Ce point de passage correspond à une température de $875^{\circ} \mathrm{C}$. et coincide exactement avec le point de transformation allotropique du fer domé par la courbe de dilatation.
Il convient de remarquer que ce n'est pas cette transformation qui peut être cause du retrait qui est constaté dans le métal, car cet état une fois établi, l'opération de dilatation donne à nouveau Ja même anomalie dans l'allongement du métal. Ce sont là des coïncidences dont on n’a pu déterminer jusqu'ici les relations.

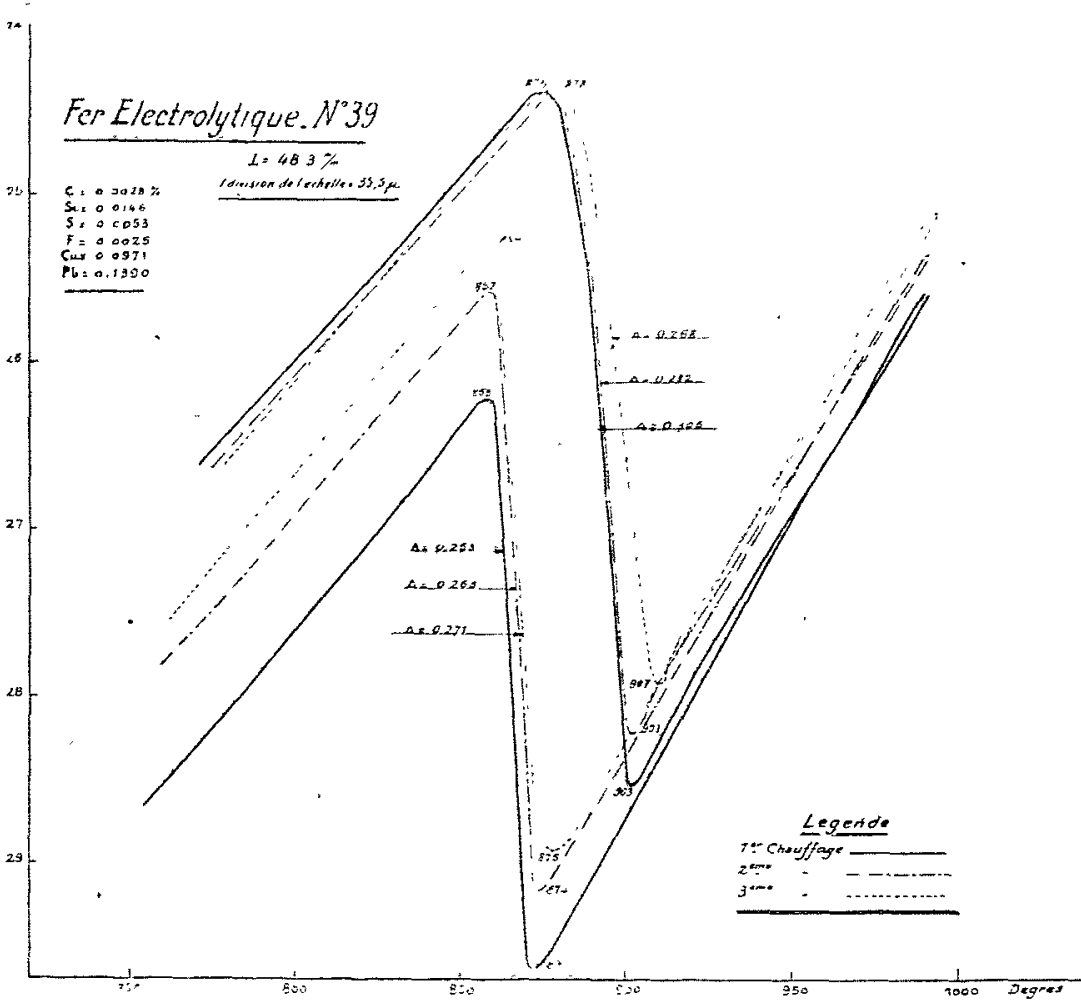

Fig. 11. - Courbes de dilatation donnant les dilatations anormales d'un fêr électrobévé pour trois chauffages successifs.

On constatera par la figure 11, que la température du point de passage est bien celle de l'anomalie de dilatation du fer électrolytique. Nous devons ajouter, a ce sujet, que le coefficient de dilatation anormàle à l'échauffement diminue à la suite de plusieurs chauffages subits par un même échantillon, tandis que le coefficient de dilatation anormale au refroidissement augmente.

Nous avons reproduit, superposés sur la figure 12, trois chauffages succesifs :

$1^{\circ}$ Chanfage : trait plein :

$$
\begin{aligned}
& \Delta \text { échauffement }=0,306 \\
& \Delta \text { refroidissement }=0,253
\end{aligned}
$$

$2^{\circ}$ Chauffage : trait mixte :

$$
\begin{aligned}
& \perp \text { echauffement }=0,282 \\
& \perp \text { refroidissement }=0,263
\end{aligned}
$$

$3^{\circ}$ Chauffage : trait pointille :

$$
\begin{aligned}
& \Delta \text { cchauffement }=0,268 \\
& \pm \text { refroidissement }=0,271
\end{aligned}
$$

Le $\perp$ moyen reste constant.

N'y aurait-il pas lieu de reverifier ces partieularites ? C'est probable, mais il nous a semblé qu’elles devaient être signalés 